\title{
Serial TSH-receptor antibody levels to guide the management of thyroid eye disease: the impact of smoking, immunosuppression, radio-iodine, and thyroidectomy
}

\author{
Jonathan C. P. Roos ${ }^{1} \cdot$ Vignesh Paulpandian ${ }^{1} \cdot$ Rachna Murthy $y^{1,2}$
}

Received: 3 October 2018 / Accepted: 4 October 2018 / Published online: 6 November 2018

(c) The Royal College of Ophthalmologists 2018

\begin{abstract}
The Thyrotropin receptor antibody (TRAb) is the main driver of Graves' disease (GD) and its most common extra-thyroidal manifestation: thyroid eye disease (TED). Though key to diagnosis, it has not been used routinely as a marker of disease activity or to guide treatment. Here we demonstrate, through a retrospective review of 105 patients with TED, that serial TRAb levels vary with time, correlate with disease activity and are affected by smoking and endocrine control. Such serial measurements can guide the modern management of thyroid eye disease, helping to prevent the more serious manifestations. We show that surgical thyroidectomy is associated with a reduction in antibody levels and a reduced rate of TED reactivation when compared to radio-iodine ablation where the stimulating antigen is not removed. This provides a molecular explanation for epidemiological studies showing radio-ablation being associated with an increased risk of orbitopathy. To demonstrate the effect of our clinical approach on a patient population, we then compared the incidence and severity of TED in a clinic in a period before and after the introduction of serial TRAb measurements. Despite an increase in disease incidence and severity at presentation over the two-decade study period, our approach saw a significant reduction in the need for surgical intervention for this orbital disorder.
\end{abstract}

\section{Introduction}

Graves' orbitopathy (GO) is an autoimmune disorder affecting the orbits and is responsible for significant morbidity and reduced quality of life [1]. Current evidence suggests the incidence of GO is declining in Europe, however, there is a paucity of epidemiological data; estimates vary from $1.6 / 100,000$ to $42 / 100,000$ in various epidemiological studies $[2,3]$.

Perhaps unique for an autoimmune disease, TED is a self-limiting inflammatory disorder, with a time course of around 2 years from onset [4]. Management decisions are therefore predicated on understanding when the disease is active and in its inflammatory stage, for this is when immunomodulatory intervention will be effective [5]. This

Rachna Murthy

rachna.murthy@ipswichhospital.nhs.uk

Ipswich Hospital NHS Trust, Ipswich, UK

2 Cambridge University Hospitals NHS Foundation Trust, Cambridge, UK period is also when any intervention likely to stimulate the disease, such as thyroid gland ablation, should be avoided to prevent exacerbation.

The most widely used tool for assessing disease activity is the clinical activity score (CAS) [6], but it has been criticised as overly simplistic, with alternative more complex scoring systems being proposed to replace it [7]. As we show elsewhere in this issue, imaging approaches by which to quantify TED, such as MRI with T2-weighted relaxation times (Das, personal communication), are emerging but not universally available [8]. CT imaging has the associated risk of radiation, making serial assessments potentially dangerous. Recently, a therapeutic study sought to use changes in proptosis as an indicator of efficacy [9]. However, proptosis has not generally been considered a reliable measure of activity or severity between patients or for individuals over time.

There remains therefore an unmet clinical need for a more sensitive marker with which to ascertain disease activity, stratify risk for individual patients as well as monitor response to treatment and to guide further interventions.

The TSH Receptor Antibody (TRAb) was discovered over half a century ago [10] and is thought to play a key role in the pathogenesis of TED. Using the most recent 
Fig. 1 Clinical activity scores at presentation plotted against initial TSH Receptor antibody levels in 105 patients attending a dedicated Thyroid Eye Clinic. Analysis shows a strong correlation between the activity score and the titre (Fisher's exact test. $\left.R^{2}=0.92 ; p<0.0001\right)$

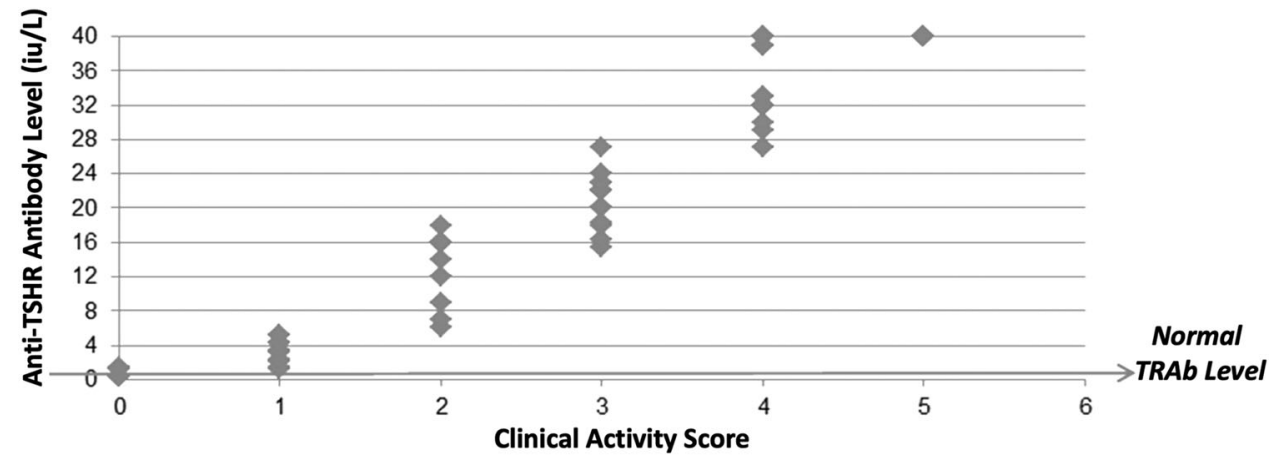

assays, thyroid-stimulating immunogloblulins (TSI) can be detected in virtually all patients with Graves' orbitopathy [11-13]. These autoantibodies are known to correlate closely with the CAS and to be predictive of the course of disease [14-17]. To determine whether they might prove a helpful biomarker of disease, we sought to study the relationship between TED activity and TRAb levels, and to determine the effect of definitive endocrine interventions and smoking status on patients' TRAb profiles over time.

We routinely test TRAb levels in every TED patient at every visit to our clinic using a standardised and commercially available kit (Elecsys ${ }^{\circledR}$ Anti-TSHR, Roche Diagnostics, Switzerland). This allowed us to conduct, retrospectively, a review of three-year serial TRAb levels in all our 105 patients seen in an 8-year period. The data was analysed by subsets of characteristics including severity, smoking status and method of definitive endocrine control.

\section{TRAb as a potential marker of activity and severity}

TRAb levels have previously been shown to be associated with disease severity and to act as a predictor of orbitopathy in most [14, 15] but not all studies [18]. To confirm such a correlation for our clinic population we plotted the TRAb levels of 105 patients as measured at first presentation against their CAS score (Fig. 1). The patients in our clinic had an average age of 52 years \pm 12.5 (range: 13-78) with a 4:1 female to male ratio. Eight patients $(8.4 \%)$ suffered with other associated autoimmune diseases and $16 \%$ had a family history of an endocrine disorder.

Figure 1 shows a strong positive correlation between initial TRAb levels and CAS score at presentation (Fisher Exact Test, $p<0.0001$ ) in keeping with the results of other studies. To evaluate other markers of disease activity we also compared MRI T2-weighted scoring and found a positive correlation with CAS scores $(p<0.0001)$ at presentation. However there was none found when comparing CAS with the level of proptosis $(p=0.37$ ) (data not shown). This suggests that the former are suitable surrogate markers for therapeutic trials, whilst proptosis should not be used to indicate response to treatment.

\section{TRAb changes over time and the effect of smoking}

Previous studies have measured TRAb levels in individual patients at variable time points during their disease and only over a limited period. Such data has then been used to extrapolate for prediction of disease course; TRAb levels below $5 \mathrm{iu} / \mathrm{L}$ are reported as conferring a significantly better chance of a benign course, compared with levels above $8 \mathrm{iu} /$ $\mathrm{L}$ where there is an up to 31 -fold increased risk of orbitopathy [14].

Many centres do not see value in measuring serial TRAbs in patients once the diagnosis is established [19]. Perhaps uniquely, we have collected serial data longitudinally on every patient over the past 8 years as part of our routine clinical work-up, spanning the time from initial presentation and diagnosis through potential definitive endocrine control and eventual disease resolution-a period usually lasting around 3 years. To determine whether serial TRAbs can act as a marker of disease activity, we plotted the results for individual patients over time. Figure $2 \mathrm{a}$ shows that in non-smokers, TRAb levels normalise with a mean of $18.5 \pm 6.5$ months, approximating to the disease course as described by the Rundle's curve.

Our patient cohort also contained $27 \%$ smokers at presentation. Smoking is recognised to increase the risk of developing orbitopathy fourfold [20] and affects its severity $[21,22]$ and response to treatment [23, 24]. We therefore analysed the results by smoking status (Fig. 2b); normalisation of TRAb levels in this group was delayed by a year with an increased average time to normalisation of $32.5 \pm$ 8.5 months. This is the first study to demonstrate prolonged persistence of TRAb in smokers with GO, hinting at a potential immunological cause for recurrence of GD and GO. It also re-enforces the importance of smoking cessation advice in patients with GO. 

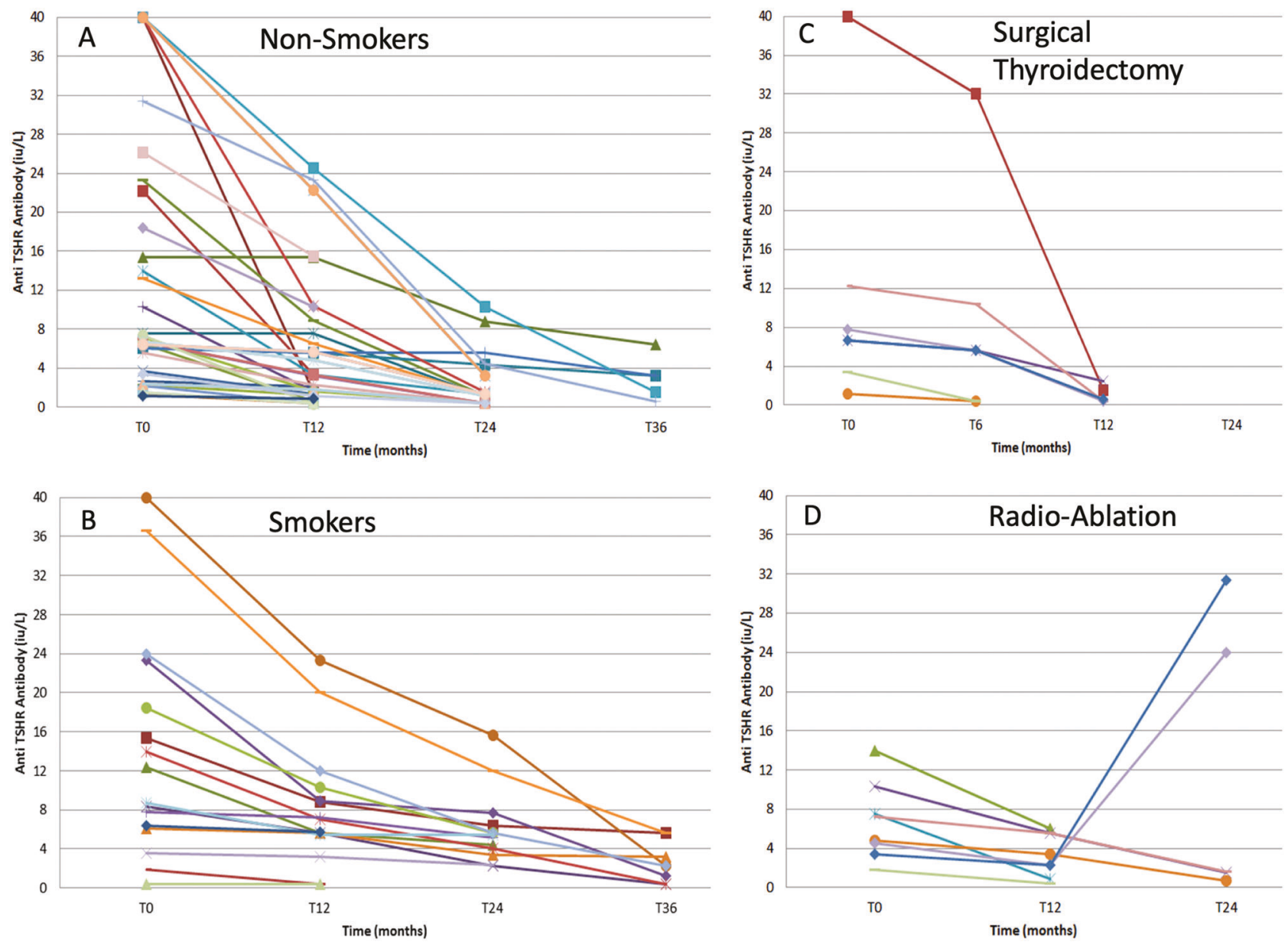

Fig. 2 a, b Serial TRAb data collected over 3 years in 105 patients separated into non-smokers (a) and the $27 \%$ who were smokers (b). Normalisation of TRAb levels was delayed by over a year in the smokers (mean time to normalisation 32 months vs 18 months for nonsmokers). c, d Serial TRAb data collected in a period immediately after definitive endocrine control with either surgical thyroidectomy in

\section{TRAb and lessons for definitive endocrine control}

In addition to smoking, another risk for worsening orbitopathy is radio-iodine ablation (RAI). 131I therapy for GD carries a risk of around $15 \%$ for development of de novo orbitopathy in the absence of steroids [25]. In contrast, a large epidemiological study covering more than 8404 patients with GD demonstrated that surgical thyroidectomy confers a very significant $(74 \%)$ reduced hazard ratio for the development of orbitopathy [26] compared to radioablation. Early thyroidectomy has also been shown in a randomised controlled trial to result in lower GO activity and a higher rate of disease inactivation [27]. In our patient cohort, a number of patients had definitive endocrine control by either surgery or RAI, giving us the opportunity to study the effect on TRAb levels.
6 patients (c) or radio-iodine ablation in 8 patients (d). Normalisation of TRAb levels occurred rapidly in the first group (7.2 \pm 3.3 months). In comparison, post-radio-iodine, the mean time was delayed to $12.6 \pm$ 6.6 months. The latter also saw an exacerbation of orbitopathy in two patients $(25 \%)$, both smokers, mirrored by a rise in their TRAb levels

Figure $2 \mathrm{c}$ shows that after surgical thyroidectomy, the six patients' TRAb levels normalised on average at 7.2 \pm 3.3 months. In comparison, in the eight patients who received RAI (Fig. 2d), the mean time was delayed by around 5 months to $12.6 \pm 6.6$ months. Furthermore, two patients $(25 \%)$ of those receiving RAI developed recurrent orbitopathy, mirrored by a rise in TRAb level despite steroid cover.

That surgical thyroidectomy with steroid cover resulted in no instances of recurrence of orbitopathy-and was coupled with a good recovery of the TRAb to normal levels - provides insight into the possible underlying pathogenic mechanism responsible for the superiority of surgical thyroidectomy seen in the epidemiological studies.

Our results also suggest an at first paradoxical management strategy for patients with thyroid orbitopathy: opting for surgical thyroidectomy even in those who are euthyroid 
Fig. 3 Serial TRAb level data collected in a single patient with Graves' disease and later orbitopathy over a period of a year. Due to progression to moderate, active TED, treatment with glucocorticoid and ciclosporin A was started 9 months into the disease and ciclosporin discontinued after 6 weeks due to intolerance of side-effects (ciclosporin treatment shown by red bar). Even during this short interval, there is a rapid and sustained reduction in the measured TRAb levels

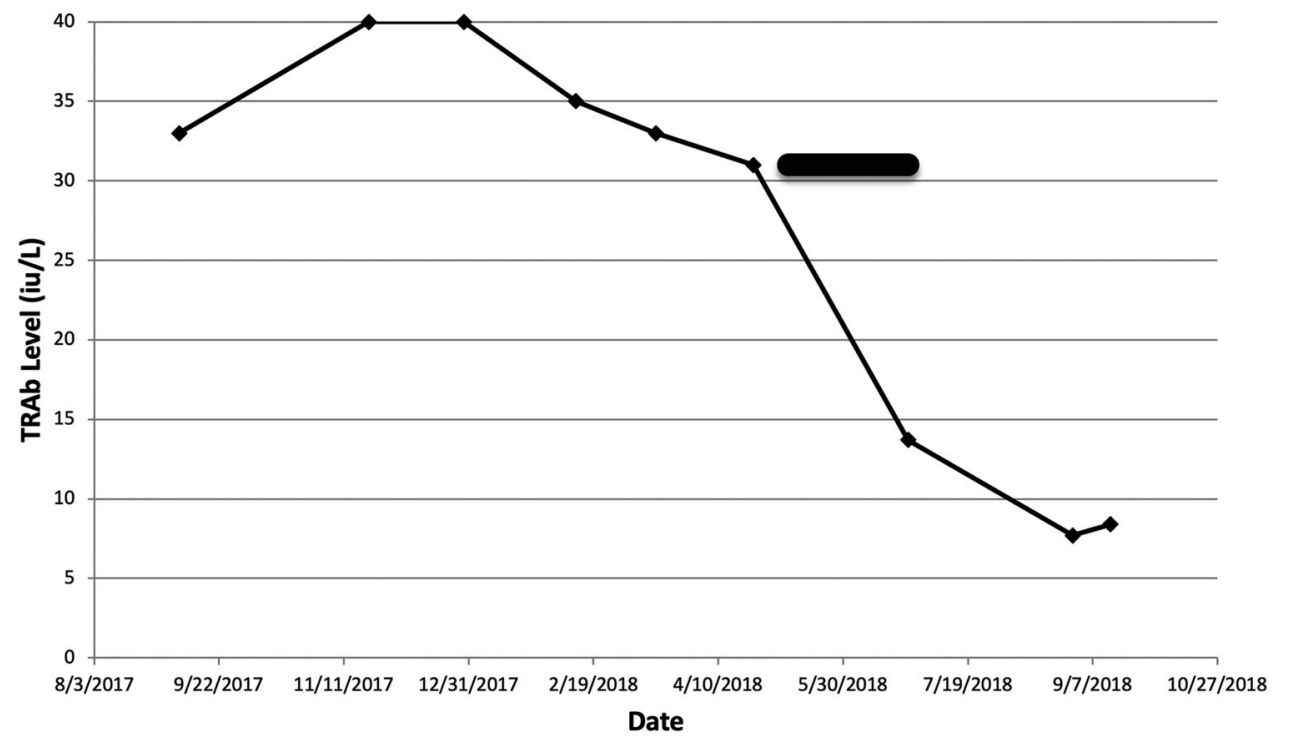

or hypothyroid. Speculatively, a reduction in the availability of TSH-R antigen after surgical removal helps to reduce the antibody drive, something which would not be expected to occur with gland destruction where antigen is likely to be released more.

\section{Treatment with ciclosporin: clinical effect of immunosuppression on TRAb}

Whilst TRAb levels can be used to chart disease progression, we hypothesised that they would also be responsive to immune-suppression and useful in monitoring the success of such treatments.

Figure 3 shows a representative example of an individual patient with active and moderately severe thyroid eye disease who was treated with low dose oral prednisolone (30 $\mathrm{mg} /$ day) and Ciclosporin A for a short period. The TRAb levels are seen to respond quickly with a significant and sustained reduction to around $8 \mathrm{iu} / \mathrm{L}$ compared to the 9 month period before, when levels remained consistently above $30 \mathrm{iu} / \mathrm{L}$. The absence of a rebound phenomenoneven with early cessation of treatment-is encouraging and has been reported previously [28].

In our clinic, we favour the use of ciclosporin $\mathrm{A}$ as a steroid sparing agent; already in 1983 it was shown to produce a rapid restoration of function with improvements in motility, visual acuity and proptosis [29]. Since then two large randomised controlled studies $[28,30]$ as well as multiple case series [31-34] have shown its benefits for a subset of TED patients. Despite its efficacy, the drug is still not widely used as it has a narrow therapeutic index (with risk of nephrotoxicity, hypertension, gum hypertrophy and hirsutism). Ideally it therefore requires a dedicated and experienced multidisciplinary team to closely monitor patients whilst on treatment.

\section{Effect of serial TRAb measurement on overall clinical outcomes}

Stepping away from the individual patient, we wondered if our approach of measuring serial TRAb levels could have an appreciable effect on the overall community we serve. We therefore performed a retrospective review of the medical records of all GO patients referred to a specialist ophthalmic service between the years of 1997 and 2016 . The mean age of the study population was $50.5 \pm 13.2$ years with a 3:1 M:F ratio. 2013 saw the introduction of a dedicated multidisciplinary GO service when serial TRAb measurements also began. In the "olden times," between 1997 to 2012, the incidence of thyroid eye disease was calculated as $2.7 / 100,000$. $49 \%$ of patients then had moderate to severe disease whilst the remaining $51 \%$ had mild disease (using the EUGOGO grading system). After the change in service provision, "modern times" from 2013 to 2016, the incidence of thyroid eye disease increased to 7.6/ 100,000 . Unexpectedly, rather than earlier detection of milder disease, patients now presented with more severe initial manifestations: $56 \%$ had moderate disease, $2 \%$ had severe and only $42 \%$ had mild disease. Despite this increase in incidence and severity, there was a significant decrease in those requiring late surgery $(p<0.005)$. We believe that being able to respond to changes in TRAb levels before worsening manifestations become apparent, or avoiding treatments for those in whom TRAbs are raised, has helped to reduce the burden of disease in our community: prevention of exacerbation, rather than cure. 


\section{Discussion}

Serial TRAb levels can inform the overall management as well as the timing of interventions in TED, reducing morbidity both at the individual and clinic population level. The modern clinical management of TED should be focussed on the prevention of complications; avoidance rather than treatment of severe orbitopathy. We suggest that serial TRAb measurements can help to guide treatment decisions and reduce the need for orbital surgery. Changing levels of autoantibody also provides a molecular underpinning for the epidemiological evidence linking smoking and RAI to worse disease. We demonstrate much prolonged persistence of TRAb in smokers with TED; and compared to RAI, thyroidectomy resulted in a faster normalisation of TRAb and no recurrences of TED.

These results complement the previous literature but have the advantage of being comprehensive; other studies have measured TRAbs in smaller groups or during a limited interval-not from the initial presentation to resolution in an entire cohort with TED as shown here. Our findings may also affect future trial design: whilst TRAb levels and MRI changes were found to correlate with clinical activity at presentation, proptosis did not, casting doubt on its applicability as a clinical or therapeutic trial marker. In TED, proptosis can occur either in active disease due to glycosaminoglycan deposition and oedema, but also secondary to adipogenesis in chronic disease [35]. Put another way: proptosis can increase as activity decreases.

The results presented are limited by the retrospective nature of the design and the fact that as a relatively small clinic running with serial measures only since 2013 means that the number of patients undergoing radio-ablation or surgical thyroidectomy in the TED cohort, is necessarily small. Furthermore, there have been significant developments in the measurement of TRAbs since that time, with stimulating and inhibitory components now frequently measured independently. Not all such tests are equally sensitive [36]. We therefore plan to repeat this study in a much larger neighbouring hospital centre where serial TRAbs have been measured consistently for longer and in a larger cohort of around 450 patients.

Overall, these data demonstrate the applicability of serial TRAb measurements for the bespoke management of individual patients. This allows for risk stratification to inform prognosis, smoking cessation, to guide thyroid gland treatment options, and monitor immunosuppression, thereby reducing morbidity. As TRAb levels have also been implicated in other thyroid-related orbitopathies, such as Hashimotos, serial measures may be helpful in the management of this condition also [37].
Acknowledgements We would like to thank Dr Clive Edelsten for valuable advice and support.

Authors contributions Dr Murthy conceived of the idea for this study. All authors have contributed to the data gathering, analysis and writing.

\section{Compliance with ethical standards}

Conflict of interest All authors are oculoplastic surgeons who manage patients with Thyroid Eye disease.

Guarantor Dr Murthy serves as guarantor of this work. It is an honest, accurate, and transparent account of the study being reported; no important aspects of the study have been omitted.

\section{References}

1. Farid M, Roch-Levecq AC, Levi L, Brody BL, Granet DB, Kikkawa DO. Psychological disturbance in graves ophthalmopathy. Arch Ophthalmol. 2005;123:491-6.

2. Abraham-Nordling M, Byström K, Törring O, Lantz M, Berg G, Calissendorff $\mathrm{J}$, et al. Incidence of hyperthyroidism in Sweden. Eur J Endocrinol. 2011;165:899-905.

3. Laurberg P, Berman DC, Bülow Pedersen I, Andersen S, Carlé A. Incidence and clinical presentation of moderate to severe graves' orbitopathy in a Danish population before and after iodine fortification of salt. J Clin Endocrinol Metab. 2012;97:2325-32.

4. Rundle FF, Wilson CW. Development and course of exophthalmos and ophthalmoplegia in Graves' disease with special reference to the effect of thyroidectomy. Clin Sci. 1945;5:177-94.

5. Dickinson AJ, Perros P. Controversies in the clinical evaluation of active thyroid-associated orbitopathy: use of a detailed protocol with comparative photographs for objective assessment. Clin Endocrinol. 2001;55:283-303.

6. Mourits MP, Koornneef L, Wiersinga WM, Prummel MF, Berghout A, van der Gaag R. Clinical criteria for the assessment of disease activity in Graves' ophthalmopathy: a novel approach. Br J Ophthalmol. 1989;73:639-44.

7. Dolman PJ. Grading severity and activity in thyroid eye disease. Ophthalmic Plast Reconstr Surg. 2018;34(4S Suppl 1):S34-S40.

8. Utech CI, Khatibnia U, Winter PF, Wulle KG. MR T2 relaxation time for the assessment of retrobulbar inflammation in Graves' ophthalmopathy. Thyroid. 1995;5:185-93.

9. Smith TJ, Kahaly GJ, Ezra DG, Fleming JC, Dailey RA, Tang RA, et al. Teprotumumab for thyroid-associated ophthalmopathy. N Engl J Med. 2017;376:1748-61.

10. Adams DD, Purves HD, Sirett NE, Beaven DW. The presence of a short-acting abnormal thyroid stimulator in the blood of a thyrotoxic patient. J Clin Endocrinol Metab. 1962;22:623-6.

11. Konuk O, \& Anagnostis. Diagnosis and differential diagnosis in Graves' Orbitopathy. In: Wiersinga M, Kahaly GJ, editors. Graves' orbitopathy: a multidisciplinary approach - questions and answers. Basel: Karger; 2017. p. 83.

12. Salvi M, Berchner-Pfannschmidt U, Ludgate M. Pathogenesis. In: Wiersinga WM, Kahaly GJ, editors. Graves' orbitopathy: a multidisciplinary approach - questions and answers. Basel: Karger; 2017. p.50.

13. Ponto KA, Kanitz M, Olivo PD, Pitz S, Pfeiffer N, Kahaly GJ. Clinical relevance of thyroid-stimulating immunoglobulins in graves' ophthalmopathy. Ophthalmology. 2011;118:2279-85.

14. Eckstein AK, Plicht M, Lax H, Neuhäuser M, Mann K, Lederbogen $\mathrm{S}$, et al. Thyrotropin receptor autoantibodies are 
independent risk factors for Graves' ophthalmopathy and help to predict severity and outcome of the disease. J Clin Endocrinol Metab. 2006;91:3464-70.

15. Lantz M, Planck T, Asman P, Hallengren B. Increased TRAb and/or low anti-TPO titers at diagnosis of graves' disease are associated with an increased risk of developing ophthalmopathy after onset. Exp Clin Endocrinol Diabetes. 2014;122: $113-7$.

16. Gerding MN, van der Meer JW, Broenink M, Bakker O, Wiersinga WM, Prummel MF. Association of thyrotrophin receptor antibodies with the clinical features of Graves' ophthalmopathy. Clin Endocrinol. 2000;52:267-71.

17. Khoo DH, Ho SC, Seah LL, Fong KS, Tai ES, Chee SP, et al. The combination of absent thyroid peroxidase antibodies and high thyroid-stimulating immunoglobulin levels in Graves' disease identifies a group at markedly increased risk of ophthalmopathy. Thyroid. 1999;9:1175-80.

18. Mukasa K, Yoshimura Noh J, Kouzaki A, Ohye H, Kunii Y, Watanabe N, et al. TSH receptor antibody titers measured with a third-generation assay did not reflect the activity of Graves' ophthalmopathy in untreated Japanese Graves' disease patients. Endocr J. 2016;63:151-7.

19. Goldberg R. $48^{\text {th }}$ Cambridge Ophthalmological Symposium: Thyroid and the Eye. 6-7th September 2018, Cambridge UK.

20. Vestergaard P. Smoking and thyroid disorders-a meta-analysis. Eur J Endocrinol. 2002;146:153-61.

21. Wiersinga WM. Smoking and thyroid. Clin Endocrinol. 2013;79:145-51.

22. Bartalena L, Pinchera A, Marcocci C. Management of Graves' ophthalmopathy: reality and perspectives. Endocr Rev. 2000;21:168-99.

23. Bartalena L, Marcocci C, Tanda ML, Manetti L, Dell'Unto E, Bartolomei MP, et al. Cigarette smoking and treatment outcomes in Graves ophthalmopathy. Ann Intern Med. 1998;129:632-5.

24. Eckstein A, Quadbeck B, Mueller G, Rettenmeier AW, Hoermann $\mathrm{R}$, Mann $\mathrm{K}$, et al. Impact of smoking on the response to treatment of thyroid associated ophthalmopathy. $\mathrm{Br} \mathrm{J}$ Ophthalmol. 2003;87:773-6.

25. Burlacu M, Daumerie C. Epidemiology. In: Wiersinga M, Kahaly GJ, editors. Graves' orbitopathy: a multidisciplinary approach questions and answers. Basel: Karger; 2017. p. 35
26. Stein JD, Childers D, Gupta S, Talwar N, Nan B, Lee BJ, et al. Risk factors for developing thyroid-associated ophthalmopathy among individuals with Graves' disease. JAMA Ophthalmol. 2015;133:290-6.

27. Meyer Zu Horste M, Pateronis K, Walz MK, Alesina P, Mann K, Schott M, et al. The effect of early thyroidectomy on the course of active Graves' orbitopathy (GO): a retrospective case study. Horm Metab Res. 2016;48:433-9.

28. Kahaly G, Schrezenmeir J, Krause U, Schweikert B, Meuer S, Muller $\mathrm{W}$, et al. Ciclosporin and prednisone v. prednisone in treatment of Graves' ophthalmopathy: a controlled, randomized and prospective study. Eur J Clin Invest. 1986;16:415-22.

29. Weetman AP, McGregor AM, Ludgate M, Beck L, Mills PV, Lazarus JH, et al. Cyclosporin improves Graves' ophthalmopathy. Lancet. 1983;2:486-9.

30. Prummel MF, Mourits MP, Berghout A, Krenning EP, van der Gaag R, Koornneef L, et al. Prednisone and cyclosporine in the treatment of severe Graves' ophthalmopathy. N Engl J Med. 1989;321:1353-9.

31. Meyer PA. Avoiding surgery for thyroid eye disease. Eye. 2006;20:1171-7.

32. Leövey A, Bakó G, Szabó J, Kálmán K, Fórizs E. Combined cyclosporin-A and methylprednisolone treatment of Graves' ophthalmopathy. Acta Med Hung. 1992;49(3-4):179-85.

33. Weissel M, Zielinski CC, Hauff W, Till P. Combined therapy with cyclosporin A and cortisone in endocrine Basedow endocrine orbitopathy: successful use in compressive optic neuropathy. Acta Med Austria. 1993;20:9-13.

34. Utech C, Wulle KG, Panitz N, Kiefer H. Immunosuppressive treatment of Graves' ophthalmopathy with cyclosporine A. Transplant Proc. 1988;20(3 Suppl 4):173-7.

35. Crisp M, Starkey KJ, Lane C, Ham J, Ludgate M. Adipogenesis in thyroid eye disease. IOVS. 2000;41:3249-55.

36. Diana T, Wüster C, Kanitz M, Kahaly GJ. Highly variable sensitivity of five binding and two bio-assays for TSH-receptor antibodies. J Endocrinol Invest. 2016;39:1159-65.

37. Kahaly GJ, Diana T, Glang J, Kanitz M, Pitz S, König J. Thyroid stimulating antibodies are highly prevalent in Hashimoto's thyroiditis and associated orbitopathy. J Clin Endocrinol Metab. 2016;101:1998-2004. 